\title{
Einleitung: Radio und Identitätspolitiken
}

\author{
Johannes MÜske, Golo FÖllmer, WALter LEIMGRUber
}

Der vorliegende Band geht auf eine Tagung des Forschungsprojekts «Broadcasting Swissness» zurück, die in Kooperation mit dem Studienkreis Rundfunk und Geschichte vom 18. bis 20. Februar 2016 an der Universität Zürich stattfand. Die vom Schweizerischen Nationalfonds geförderte Forschungsgruppe von Universität Zürich, Universität Basel, Hochschule Luzern und weiteren Projektpartnern erforschte die Konstruktion und Vermittlung von «Swissness〉 mittels (Volks-) Musik. ${ }^{1}$ Am Beispiel einer Tonbandsammlung des Schweizer Auslandsradios untersuchte das Projekt die musikalische Populärkultur von der Nachkriegszeit bis in die Gegenwart aus musikforschender, kulturanthropologischer und ethnomusikologischer Perspektive. ${ }^{2}$ Der englisch klingende Neologismus «Swissness〉

1 Im Zentrum des Projekts stand der Schweizerische Kurzwellendienst (KWD, ab 1978 Schweizer Radio International), der als Auslandsradio von 1935 bis 2004 〈Swissness〉 in der Welt verbreitete und heute im Internet unter Swissinfo.ch über die Schweiz berichtet. Die internationale Tagung war zugleich die Abschlusstagung der ersten Phase des Projekts, das insgesamt vier Jahre (12/2012-11/2015 und 12/2015-11/2016) mit Förderung des SNF im Rahmen des «Sinergia»-Programms lief; Informationen zum Projekt: Müske/Oehme-Jüngling/Hengartner/Leimgruber (2017) sowie unter https:// www.isek.uzh.ch.

2 Der Bestand Volksmusik oder die Sammlung Dür, benannt nach dem Gründer und damaligen Leiter der «Sonothek» des Kurzwellendienstes Fritz Dür, umfasst gut 7600 1/4"-Tonbänder, die zwischen 1957 und 1967 zusammengetragen wurden. Es handelt sich um zeitgenössische Volks- und Populärmusik, die auf Industrietonträgern kaum erhältlich war und daher von der SRG SSR schweizweit produziert wurde, beispielsweise mit regionalen Musikgruppen und den Radio-Orchestern. Mit Glück und Umsicht konnte der Bestand erhalten werden. Die Sammlung Dür ist Eigentum der SRG 
ist seit den 1990er Jahren als Set von positiv konnotierten Vorstellungen und Werten wie Qualität, Verlässlichkeit oder Heimat für die Vermarktung von Schweizerischem populär geworden; die Forschungsgruppe hat sich den Begriff geliehen, um die populärkulturelle Dimension der Konstruktion und Verbreitung von schweizerischer Identität zu betonen.

Die Leitfrage des gesamten Projektes war, wie und vor welchen gesellschaftlichen wie institutionellen Hintergründen sich populärmusikalisches Schaffen mit der Institution Rundfunk zu einer wirkmächtigen Stimme zur Verbreitung von - klingender - «Swissness〉 etablieren konnte. Damit nimmt das Projekt eine Perspektive auf, welche die soziale Bedeutung von populärer Musik für Gruppen und damit Identitätsfragen als zentrales Forschungsthema erschließt (Frith 1996). ${ }^{3}$ Das Radio spielte seit seiner Verbreitung als Alltagsmedium in den 1920er Jahren in vielen europäischen Ländern eine zentrale Rolle bei der Verbreitung von kulturellen Werten. Die Einführung erläutert daher zuerst knapp das kulturwissenschaftliche Konzept der Identität. Anschließend werden grundlegende Aspekte des Radios als kulturpolitischer Institution skizziert und schließlich die Beiträge des Bandes vorgestellt.

\section{WER BRAUCHT «IDENTITÄT»?}

«Wer braucht 〈Identität»?», könnte einmal mehr gefragt und darauf verwiesen werden, dass dieses komplexe Konzept bereits in den 1990er Jahren wissenschaftlich eingehend diskutiert und dekonstruiert wurde (z.B. Hall 1996). Doch habe die Dekonstruktion eines essentialistischen Identitätsbegriffs, so der Soziologe Stuart Hall, weder dazu geführt, dass «Identität» als wissenschaftlich unbrauchbarer Begriff verworfen wurde, noch dazu, dass er als Postulat den politischen Alltagsdiskurs verlassen hätte. Gerade in den 1990er Jahren war vielmehr

und wird seit 1997 in Bern von der Schweizer Nationalphonothek aufbewahrt. Der Bestand wurde in transdisziplinärer Zusammenarbeit mit den Projektpartnern SRF, Nationalphonothek und Memoriav digitalisiert, in den Metadaten verbessert und erklingt wieder regelmäßig im Radio. Zur Projektgenese und Überlieferungsgeschichte der Sammlung Dür vgl. die Beiträge von Rudolf Müller und Hans-Rudolf Dürrenmatt in Hengartner/ Müske et al./Forschungsgruppe Broadcasting Swissness (2016).

3 «But popular music is popular not because it reflects something or authentically articulates some sort of popular taste or experience, but because it creates our understanding of what (popularity) is, because it places us in the social world in a particular way.» (Frith 1996: 121). 
eine «diskursive Explosion» (Hall 1996: 1) rund um das Konzept zu verzeichnen. Seitdem hat sich der Diskurs hin zum begrifflichen Zwilling der Identitätspolitiken verschoben, womit die strategische Nutzung von Identität bzw. Identitätsmarkern bezeichnet wird. Politiken der Identität kamen in den 1970er Jahren im Umfeld der US-amerikanischen politisch-kulturellen Bewegungen auf (neue soziale Bewegungen, z.B. Civil Rights, LGBT, American Indian movements), verstanden wurden darunter die politischen Aktivitäten und Praktiken marginalisierter Gruppen in ihrem Kampf gegen Ungleichheit und für gesellschaftliche Anerkennung. ${ }^{4}$ Kulturanthropologische Forschungen mit ihrem Fokus auf Akteur_innen, Praktiken und Interessen, kurz: soziale und kulturelle Faktoren, haben daran einen wichtigen Anteil (Biddle 2012: xvi-xix), einhergehend mit einem 〈weiten〉 Politik-Begriff. ${ }^{5}$

Identitätspolitiken können über den Fokus auf neue soziale Bewegungen hinaus verstanden werden als «any mobilization related to politics, culture, and identity» (Bernstein 2005: 48). Identität ist ein komplexes Konzept, das in unterschiedlichsten Alltagssphären genutzt wird und wirksam ist. ${ }^{6}$ Generell sind Geschichte und Kulturerbe der Gegenstand konkurrierender Interessen (vgl. Biddle 2012: xxii). Berücksichtigt man die Vielschichtigkeit von Identitätskonzepten und politischen Akteur_innen, so sind Identitätspolitiken stets auch auf staatlicher und nationaler Ebene zu beobachten. Lanciert werden etwa nationale Feier-

4 Als Identitätspolitiken können die politischen Maßnahmen gefasst, die Ungleichheiten sichtbar machen, gesellschaftliche Aufmerksamkeit für die jeweilige Ungleichbehandlung oder Stigmatisierung schaffen und an der Behebung dieser sozialen und kulturellen Ungerechtigkeiten mitwirken (vgl. z.B. Heyes 2002, Wiarda 2016: 147ff.). Allerdings, so auch die Kritik an den neuen sozialen Bewegungen, würden diese den Fokus ihrer identitätspolitischen Aktivitäten nicht auf die Beseitigung sozialer Missstände legen, sondern die Auseinandersetzung auf das Gebiet des Symbolischen verlagern und damit die eigentlichen gesellschaftlichen Probleme nicht adressieren (vgl. z.B. Heyes ebd.; Bernstein 2005: 49-50. Kritiker_innen der Identitätspolitiken merkten zudem an, dass diese letztlich auf essentialistischen Kulturbegriffen beruhten und bestimmte Identitätsmarker von Minoritäten, etwa im Hinblick auf 〈Rasse〉, 〈Geschlecht〉 oder ethnische Konzepte, eindimensional festschreiben würden.

5 Beispielhaft zeigt sich dieses breite ethnografisch gefärbte Politik-Verständnis und das damit einhergehende Forschungsinteresse in der empirisch-kulturwissenschaftlichen Forschung, wo «es um das konkrete Handgemenge der Artikulation, der Aushandlung und der Praktiken von Politik geht» (Schönberger 2018: 35; m. w. Nachweisen).

6 Der britische Soziologe Richard Jenkins nennt z.B.: personal identity, life-style, social position and status, politics, bureaucracy and citizenship (2000: unpag. [2f.]). 
tage, zu denken ist ebenso an Denkmäler und die vielfältige Pflege des kulturellen Erbes in den europäischen Nationalstaaten seit dem 19. Jahrhundert. Die Historiker Hobsbawm und Ranger (1983) sprechen von «erfundenen Traditionen», um die Aktivitäten zu beschreiben, mit denen die Entstehung solcher Kulturen gefördert werden soll. Kanonisierende Sammlungen wie die Lieux de mémoire (Nora 1984ff.), die zahlreiche Nachfolgewerke in vielen Ländern inspiriert haben, darunter auch in der Schweiz (Kreis 2010), überblicken diese Kultur- und Geschichtspolitiken auf eindrückliche Weise und sind ihrerseits selbst ein Teil davon. Kern der kulturpolitischen Maßnahmen mit Bezug zur Vergangenheit ist stets die Stärkung einer gemeinsamen Gruppenidentität, d.h. die Promotion eines Sets von kollektiv geteilten Erzählungen und Werten. ${ }^{7}$ Diese «kulturale Dimension von Identität» (Bausinger 1977) gilt es weiterhin zu erforschen, denn Identität als gefordertes gesellschaftliches Ideal ist weiterhin relevant - «Identitätsangebote aufzuspüren» und «Vorstellungen bruchloser Identität zu relativieren» (ebd.: 215) ist Aufgabe einer empirischen Kulturwissenschaft, die Alltagsphänomene in ihrer Komplexität und geschichtlichen Gewordenheit untersucht. ${ }^{8}$

\section{RADIO UND IDENTITÄTSPOLITIKEN}

Seit Beginn des zivilen Rundfunks wurde das Radio von staatlicher Seite als kulturpolitische Institution behandelt und gefördert und in den 1920er und 30er Jahren in vielen Ländern aus diesem Grund nationalisiert oder zumindest staatlich reguliert. Die von Vereinen, Genossenschaften und Unternehmen gegründeten Radiosender wurden beispielweise in der Schweiz 1931 zur Schweizerischen Rundspruch-Gesellschaft unter einem Dach zusammengeführt, in der die «Studios» weiterhin unabhängig voneinander agierten, aber qua Rundfunkkonzession einen gesetzlich festgelegten Programmauftrag zu erfüllen hatten. Die Program-

7 Clifford Geertz' berühmte Definition von Kultur ist in diesem Zusammenhang weiterhin aktuell: «The cultures of different countries are very much a part of their identity politics. [...] Culture, following anthropologist Clifford Geertz is a shared system of meaning that people use to make sense of the world. [...] it is a deeply rooted set of values and beliefs, and ways of behaving. Culture provides a group or a country with its identity» (Wiarda 2016: 151).

8 Mit Stuart Hall (1996: 16): Die Untersuchung und Theoretisierung «of identity is a matter of considerable political significance, and is only likely to be advanced when both the necessitiy and the <impossibility> of identities, and the suturing of the psychic and the discursive in their constitution, are fully and unambiguously acknowledged.» 
me der Studios wurden gebündelt und über drei Landessender bei Sottens, Beromünster und Monte Ceneri ausgestrahlt, je für die französische, deutsche und italienische Schweiz, sodass die Sender schon früh «nicht einseitigen, lokalen Landesinteressen dienen» sollten; vielmehr sollte die Neuorganisation «ein entschiedener Schritt vom lokalen Radiobetrieb zum grosszügigen schweizerischen Rundspruch sein, der mit anderen Ländern zum mindesten konkurrieren kann und so das Interesse der Hörer stimuliert» (SRG Jahrbuch 1931: 9). ${ }^{9}$

Sehr deutlich wird dieses Ziel in der kulturpolitischen Doktrin der «Geistigen Landesverteidigung», die in der Schweiz in den 1930er Jahren als vielschichtiges Maßnahmenbündel entstand. Sie wurde getragen von unterschiedlichen staatlichen Ebenen und staatsnahen Institutionen und hatte das Ziel, angesichts der Bedrohungen aus dem Norden und Süden und parallel zur militärischen und wirtschaftlichen Landesverteidigung, auch auf kulturellem Gebiet die Zusammengehörigkeiten über die Sprachgrenzen der Schweiz hinweg zu vermitteln und so das Nationalitätsgefühl zu stärken. Neben der staatlichen Förderung von Literatur, Filmen und Ausstellungen, die das «Schweizertum» propagierten, förderte die Bundesregierung auch das Radio als neues Medium, da man davon ausging, dass das Radio am besten breiteste Bevölkerungskreise - unabhängig vom Bildungsgrad und auch an abgelegenen Orten - erreichen könne: «Im Laufe des letzten Jahrzehntes hat sich das Radio zum wichtigsten und machtvollsten Kultur- und Propagandawerkzeug ausgewachsen», führte der Schweizerische Bundesrat (1938: 1005) in den zentralen kulturpolitischen Leitlinien aus («Kulturbotschaft»). ${ }^{10}$ Innerhalb der nationalstaatlichen Grenzen sollte das Radio einen Bildungsauftrag erfüllen, schweizerische Werte propagieren und so zum Zusammenhalt des Gemeinwesens beitragen. ${ }^{11}$

Die Gründung des Kurzwellendienstes innerhalb der SRG im Jahr 1938 (nach einer Phase des Probebetriebs ab 1935) verdankt sich der Idee dieser kulturpolitischen Doktrin. In dieser Zeit erfährt die «Kulturwahrung» ihren Höhepunkt, und auch die «Kulturwerbung» im Ausland wird als Ziel der nationalen Identitätspolitiken festgeschrieben. Dem Radio sind diese beiden Aufgaben zugedacht - insbesondere der Kurzwellendienst als Botschafter der Schweiz im

9 Zur Geschichte des Schweizerischen Rundfunks vgl. insb. Schweizerische Radio- und Fernsehgesellschaft et al. (2000, 2006, 2012).

10 Der Bundesrat erarbeitet in regelmäßigen Abständen solche kulturpolitischen Leitlinien (Kulturbotschaft), zuletzt im Jahr 2015 (für 2016-2020).

11 Einen Bezugspunkt für einige Beiträge in diesem Band bilden die Überlegungen zu Radio und der imagined community von Michelle Hilmes (2012/1997), in Anknüpfung an das Konzept von Benedict Anderson. 
Ausland wird ausdrücklich genannt. Sinnfällig wird dies in einer zentralen Passage in der Kulturbotschaft, die den Auftrag des Auslandsradios programmatisch umreißt:

«Wir müssen, ohne aufdringlich oder überheblich zu werden, dem Ausland zeigen, dass wir nicht nur ein Land der Industrie, des Handels und des Fremdenverkehrs sind, dass die Schweiz vielmehr auch ein Land ist von hoher Kultur, von alter, bodenständiger und eigenartiger Zivilisation, und dass wir zu allen Zeiten unsern eigenwertig-schweizerischen Beitrag an die Gesamtkultur Europas und der Welt geleistet haben.» (Schweizerischer Bundesrat 1938: 1011) $)^{12}$

Wie allen internationalen Radios jener Zeit fiel auch dem Kurzwellendienst die Aufgabe zu, für die eigene Bevölkerung im Ausland («fünfte Schweiz» genannt, in Ergänzung zu den vier Sprachregionen) sowie für interessierte Kreise im Ausland attraktive Programme mit Nachrichten und landeskundlichen Informationen in den Äther zu schicken. ${ }^{13}$ Die betreibenden Nationen erhofften sich von ihren Kurzwellenradios, insbesondere in Zeiten des Kalten Krieges, positive Effekte in Bezug auf die internationale Vermittlung der eigenen politischen Botschaften (Badenoch et al. 2013) sowie Wirtschafts-, Tourismus und Standortförderung (Programme des Schweizer Auslandsradios wurden beispielsweise auch im Swissair In-Flight Entertainment gesendet).

\section{RADIO, POPULÄRE MUSIK UND SOUND - KUlturWissenschaftliche Perspektiven}

Die in diesem Band versammelten Beiträge behandeln das Thema Radio und Identitätspolitiken aus unterschiedlichen disziplinären Perspektiven. Die kulturwissenschaftliche Forschung zu Klängen bildet einen Schwerpunkt innerhalb des interdisziplinären Forschungsinteresses zur sinnlichen Dimension der Alltagswelt. Dies hängt zweifellos mit der medialen Speicherbarkeit von Klängen zusammen, womit seit der Erfindung des Phonographen vor gut 140 Jahren die An-

12 Dieser Auszug wurde von den Radioschaffenden immer wieder zitiert, um die Arbeit des Kurzwellendienstes zu legitimieren, etwa in den Jahresberichten der SRG für die Jahre 1957 und 1958.

$13 \mathrm{Zu}$ Programm und Geschichte von KWD bzw. SRI vgl. die Beiträge der Forschungsgruppe in Hengartner/Müske et al./Forschungsgruppe Broadcasting Swissness (2016). 
lage von umfassenden Quellenkorpora in Rundfunkarchiven, Forschungs- und Kulturerbe-Institutionen möglich wurde. ${ }^{14}$

Die Beiträge in diesem Band sind nach verschiedenen thematischen Schwerpunkten geordnet und greifen verschiedene Schwerpunktsetzungen innerhalb der Sound Studies auf. Ein gewisser zeitlicher Fokus liegt auf dem mittleren Drittel des 20. Jahrhunderts und ist thematisch bedingt, da in diese Zeit die goldene Ära des weltweiten Kurzwellenfunks wie auch des Radios generell fällt. Auftakt bilden zwei Hauptvorträge, die beide die zweite Hälfte des 20. Jahrhunderts in den Blick nehmen. CHRISTINE BURCKHARDT-SEEBASS geht in einem sehr persönlichen Rückblick den Wellenbewegungen der Popularmusikpraxis und -pflege in verschiedenen Alltagskontexten nach, darunter auch Wissenschaft und privates Musizieren, wobei sie insbesondere die Vereinnahmungen und Emanzipationen von Volksmusik in den Blick nimmt. Die zweite Keynote, von MORTEN MiCHELSEN, untersucht die strukturelle Einbettung des Radios in den Alltag und die Zusammenhänge von Radioklängen, -programmen und kulturellem Wandel mit dem Schwerpunkt auf der gesellschaftlichen Modernisierungsphase vor 1960.

Der erste Themenschwerpunkt geht auf das Radio als klingendes Medium ein. Die Beiträge sind vor allem medienwissenschaftlich informiert; zentral ist die spezifische Medialität des Radios, in der bearbeitete und unbearbeitete Originaltöne, gesprochenes Wort, Musik, Geräusche und Sounddesign sinnlich inszeniert werden. Mit der Gestaltung der medialen Klangwelt sollen ganz unterschiedliche Ziele erreicht werden, etwa das Evozieren von Stimmungen oder das Authentisieren von Situationen. ${ }^{15}$ HANS-UlRICH WAGNER untersucht die Konstruktion von Räumen durch Medien am Beispiel der Stadt Hamburg, begriffen als ein Raum, der durch kommunikative Bezugnahmen immer wieder neu hergestellt und verhandelt wird. In seinem Beitrag stellt er ein mehrschrittiges Analysemodell klingender Quellen vor. Auch der Beitrag von FELIX WIRTH geht auf

14 Mit der Erforschung der klingenden bzw. sinnlichen Dimension der Alltagswelt schreiben sich Projekt und Tagung in die interdisziplinären Forschungsfelder Sound Studies und Anthropology of the Senses ein, zu denen in den letzten Jahren verschiedene Forschungsprojekte, Zeitschriften, Buchreihen und programmatische Sammelbände entstanden sind. Vgl. einführend z.B. Bendix (2000), Classen (1997), Pinch/ Bijsterveld (2012), Sterne (2012).

15 Auch geht es um die Erkennbarkeit der Inhalte (z.B. die ausführende Beschreibung von Situationen oder die Unterlegung von Gesprochenem mit passenden Geräuschen) sowie um die Wiedererkennbarkeit von Sendern als Marken; vgl. zur methodischen und theoretischen Annäherung an die Untersuchung der Ästhetik des Radios z.B. Föllmer (2013). 
die klingende Dimension des Radios anhand von historischen Quellen ein; erforscht werden am Beispiel des Deutschschweizer Radios die klanglichen Praktiken, um Science Fiction bzw. imaginative Zukunftsvorstellungen darzustellen. Einen theoretischen Zugang zu dieser Fragestellung wählt ROLAND JÄGER, der auf der Grundlage linguistischer Literatur und am Beispiel einer Quelle ein Konzept der «auditiven Transkriptivität» entwickelt, das erklärt, wie im Hörfunk strategisch Bedeutungen auditiv konstruiert werden.

Ein weiterer Schwerpunkt behandelt das Radio und seine Bedeutung für die Konstruktion von imagined communities, wobei die konzeptionellen Überlegungen Hilmes' (2012/1997) in Anknüpfung an Benedict Andersons Begriff einen wichtigen Bezugspunkt der Beiträge bilden. KATHRIN DRECKMANN zeigt in ihrer Arbeit zu den Wunschkonzerten im Nationalsozialismus, wie das Radio zu einem Baustein der Manipulation wurde und zur Herstellung einer «Volksgemeinschaft» beitrug. Nach dem Zweiten Weltkrieg, insbesondere mit der Einführung des UKW-Radios, stellten Radiosendungen vermehrt die regionale Identität in den Mittelpunkt, wie THOMAS FELFER in seinem Beitrag zu Glockensendungen im Österreichischen Radio herausarbeitet. Auch im Konflikt soll das Radio Gruppenidentitäten stärken: MARTINA NOVOSEL untersucht in ihrem Beitrag, wie das Radio im Kroatienkrieg traditionelle Tamburica-Musik sendete, die als besonders〈kroatisch〉 galt, um die Moral der Bevölkerung und der Truppen zur stärken.

Die Beiträge des dritten, ethnomusikologischen Schwerpunkts sind im Projektzusammenhang von Broadcasting Swissness entstanden und thematisieren die Konstruktion und Vermittlung von Heimat durch das Radio. THOMAS JÄRMANN behandelt die Frage, wie das Radio «Heimatgefühle» mit Hilfe von symphonischer Blasmusik wecken wollte - die Autoren der beiden Werke, die er exemplarisch untersucht, hatten 〈Swissness〉 in Form von populären Volksliedmelodien in ihre Kompositionen eingeflochten. Die Beiträge von JOHANNES RÜHL und JOHANNES MÜSKE erforschen beide Aushandlungen in den 1950er und 60er Jahren im Zusammenhang mit dem Rundfunk darüber, was die «echte» Volksmusik ausmache. Rühl geht es um in der Schweiz medial diskutierte Definitionsfragen, wie die «echte»Volksmusik vom «Kitsch» abzugrenzen sei, während Müske die Zusammenarbeit von Volkskunde und Radio untersucht, deren gemeinsame Tagungen nicht zuletzt aufgrund von ungelösten Definitionsfragen einschliefen.

Das Radio als Medium der Bildung und des Kulturaustauschs wird im anschließenden rundfunkhistorischen Abschnitt untersucht. Der Beitrag von ANDREAS ZEISING über die Radiosendungen zum Dürer-Jubiläum 1928 rückt das Radio als Medium «nationaler〉 Identitätsarbeit im Bildungskontext ins Blickfeld. 
Der Beitrag verdeutlicht, wie präsent der Belehrungsgedanke in den frühen Radioprogrammen war; enge Bezüge zeigen sich zum Abschnitt zur Konstruktion von imaginierten Gemeinschaften, denn unverkennbar enthalten die Bildungssendungen jener Zeit starke nationalistische Töne. Ebenfalls das Radio als Vermittler kultureller Werte behandelt der Beitrag von RÜDIGER RITTER. Die JazzProgramme im internationalen amerikanischen Radio während des Kalten Krieges zeugten von amerikanischem Sendungsbewusstsein und sollten das Beste der amerikanischen Musik vermitteln, dabei gaben sie sich andererseits unpolitisch so konnten sie den kulturellen Austausch zwischen den durch den Eisernen Vorhang getrennten Blöcken fördern.

Schließlich wird das Radio in historischer Perspektivierung als soziales Medium untersucht. Zentral ist das Ziel der Radiosender, für ihre Hörer_innen ein Angebot zu schaffen, mit dem diese sich identifizieren. FANNY JONES erforscht in ihrem Beitrag die Interaktion des Schweizer Auslandsradios mit seinem Publikum weltweit, lange bevor es die heutigen sogenannten sozialen Medien gab. Es wird deutlich, dass etwa Hörerbindung, die Ansprache unterschiedlicher Hörergruppen über das Programm sowie Wissen über die Zusammensetzung des eigenen Publikums zu generieren, schon in den 1950er Jahren wichtige Zielsetzungen der Hörerforschung waren. Sie stellen bis heute das Radio vor Herausforderungen, wie THOMAS WILKE am aktuellen Beispiel des südwestdeutschen Formatradios SWR3 und seines Umgangs mit sozialen Medien (Facebook) untersucht. Hier zeichnen sich neue Strategien der Hörerbindung und neue Kommunikationsformen mit der Sender-Community ab, welche die Redaktionen aber auch vor Herausforderungen stellen, etwa in Bezug auf die nachträgliche Regulierung von geposteten Beiträgen, die ja nicht mehr zuerst eine Hörerbriefredaktion durchlaufen.

Die Forschungsperspektiven in diesem Band behandeln das Radio als identity technology (Poletti/Rak 2014) nicht erschöpfend; stärker gegenwartsbezogene und ethnografische Perspektiven etwa könnten Online- und Community-Radios und konvergierende Medien in den Blick nehmen sowie die Frage aufgreifen, wie kulturwissenschaftliche Klangforschung auch medial angemessen repräsentiert werden kann (vgl. z.B. Feld/Brenneis 2004).

In der Gegenwart scheint in besonderer Deutlichkeit eine dunkle Seite der Politiken der Identität auf. In vielen - auch demokratischen - Ländern versuchen interessierte Akteur_innen, Medien für ihre Ziele zu nutzen, kritische Medien als unglaubwürdig zu verunglimpfen oder gar in ihrer Arbeit zu behindern. Diese besorgniserregenden Entwicklungen verweisen auf das anhaltende politische Aktivierungspotenzial von Identitätsangeboten. Gleichzeitig besteht die Gefahr, dass die identitätspolitischen Auseinandersetzungen von anderen wichtigen ge- 
sellschaftlichen Problemfeldern - Umwelt, Soziales etc. - ablenken. Die Beiträge in diesem Band verdeutlichen, dass kulturelle Identitäten etwas Spekulatives und Imaginäres, etwas Fließendes bleiben und sich stets im Modus des Werdens und in konstanter gesellschaftlicher Diskussion befinden. Die kulturwissenschaftliche Forschung erinnert daran, dass Vorsicht geboten ist, sobald es um Identität geht.

\section{DANK}

Die Herausgeber danken den Autorinnen und Autoren herzlich für ihre Bereitschaft, ihre Tagungsbeiträge für den Band zur Verfügung zu stellen, ebenso unseren Lektoren und dem transcript Verlag für die Begleitung der Publikation. Wir danken zudem dem Kooperationsnetzwerk unserer Forschungsgruppe für die hervorragende Zusammenarbeit, insbesondere bei der Zugänglichmachung der Sammlung Dür für eine breite Öffentlichkeit. Das Projekt Broadcasting Swissness wurde großzügig vom Schweizerischen Nationalfonds gefördert. Viel zu früh verstarb nach schwerer Krankheit Thomas Hengartner, Leiter der Forschungsgruppe, inspirierender Kollege, Mentor und Freund. Seinem Andenken ist dieser Band gewidmet.

\section{LITERATUR}

Badenoch, Alexander; Andreas Fickers; Christian Henrich-Franke, Hg. (2013): Airy Curtains in the European Ether. Broadcasting and the Cold War. Baden-Baden: Nomos.

Bausinger, Hermann (1977): Zur kulturalen Dimension von Identität. In: Zeitschrift für Volkskunde 73 (2), 210-215.

Bendix, Regina (2000): The Pleasures of the Ear. Toward an Ethnography of Listening. In: Cultural Analysis 1, 33-50.

Bernstein, Mary (2005): Identity Politics. In: Annual Review of Sociology 31, 47-74.

Biddle, Ian (2012): Music and Identity Politics (Introduction). In: Ders., Hg.: Music and Identity Politics. London/New York: Routledge, $\mathrm{xi}-\mathrm{xxiv}$.

Classen, Constance (1997): Foundations for an Anthropology of the Senses. International Social Science Journal (UNESCO) 49 (153), 401-412.

Feld, Steven/Donald Brenneis (2004). Doing Anthropology in Sound. In: American Ethnologist 31 (4), 461-474. 
Föllmer, Golo (2013): Theoretisch-methodische Annäherungen an die Ästhetik des Radios. In: Axel Volmar/Jens Schröter, Hg.: Auditive Medienkulturen. Techniken des Hörens und Praktiken der Klanggestaltung. Bielefeld: transcript, 321-337.

Frith, Simon (1996): Music and Identity. In: Stuart Hall/Paul du Gay, Hg.: Questions of Cultural Identity. London etc.: Sage, 108-127.

Hall, Stuart (1996): Introduction: Who Needs «Identity»? In: Ders./Paul du Gay, Hg.: Questions of Cultural Identity. London etc.: Sage, 1-17.

Hengartner, Thomas/Johannes Müske et al./Forschungsgruppe Broadcasting Swissness, Hg. (2016): Die Schweiz auf Kurzwelle. Musik - Programm Geschichte(n). Zürich: Chronos.

Heyes, Cressida (2002): Identity Politics. In: The Stanford Encyclopedia of Philosophy (Fall 2018 Edition), hg. von Edward N. Zalta, https://plato.stanford. edu/archives/fall2018/entries/identity-politics/, 1.10.2018.

Hilmes, Michelle (2012): Radio and the Imagined Community. In: Jonathan Sterne, Hg.: The Sound Studies Reader. London/New York, 351-362 (zuerst 1997).

Hobsbawm, Eric J./Terence O. Ranger, Hg. (1983): The Invention of Tradition. Cambridge: Cambridge University Press.

Jenkins, Richard (2000): The Limits of Identity: Ethnicity, Conflict and Politics. In: Sheffield OnLine Papers in Social Research (2, Nov. 2000), https:// www.sheffield.ac.uk/polopoly_fs/1.71398!/file/RJenkins_publications.pdf, 1.9.2018.

Kreis, Georg (2010): Schweizer Erinnerungsorte: Aus dem Speicher der Swissness. Zürich: NZZ Libro.

Müske, Johannes/Karoline Oehme-Jüngling/Thomas Hengartner/Walter Leimgruber (2017): Broadcasting 〈Swissness〉. Die klingende Schweiz im Radio kulturwissenschaftliche Perspektiven auf auditive Identitätspolitiken mit Schweizer Klängen. In: Karl Braun/Claus-Marco-Dieterich et al., Hg.: Kulturen der Sinne: Zugänge zur Sensualität der sozialen Welt. 40. Volkskundekongress, Zürich 2015. Würzburg: Königshausen \& Neumann, 429-435.

Nora, Pierre, Hg. (1984-1992): Les Lieux de Mémoire. 7 Bde. Paris: Gallimard.

Pinch, Trevor/Karin Bijsterveld (2012): New Keys to the World of Sound. In: Dies., Hg.: The Oxford Handbook of Sound Studies. Oxford: Oxford University Press, 3-35.

Poletti, Anna/Julie Rak, Hg. (2014): Identity Technologies. Constructing the Self Online. Madison: University of Wisconsin Press.

Schönberger, Klaus (2018): Zur Spezifik des Politischen in der Empirischen Kulturwissenschaft. In: Johanna Rolshoven/Ingo Schneider, Hg.: Dimensio- 
nen des Politischen. Ansprüche und Herausforderungen der Empirischen Kulturwissenschaft. Berlin: Neofelis, 35-50.

[SRG] Schweizerische Rundspruch-Gesellschaft (1931): Erster Jahresbericht. Mit Betriebsrechnung und Bilanz per 31. Dezember 1931 und Budget pro 1932. Bern: SRG SSR.

Schweizerische Radio- und Fernsehgesellschaft/Markus T. Drack/Therese Egger, Hg. (2000): Radio und Fernsehen in der Schweiz. Geschichte der Schweizerischen Rundspruchgesellschaft SRG bis 1958. Baden: hier + jetzt.

Schweizerische Radio- und Fernsehgesellschaft/Theo Mäusli/Andreas Steigmeier, Hg. (2006): Radio und Fernsehen in der Schweiz: Geschichte der Schweizerischen Radio- und Fernsehgesellschaft SRG 1958-1983. Baden: hier + jetzt.

Schweizerische Radio- und Fernsehgesellschaft/Theo Mäusli/Andreas Steigmeier/François Vallotton, Hg. (2012): Radio und Fernsehen in der Schweiz. Geschichte der Schweizerischen Radio- und Fernsehgesellschaft SRG 19822011. Baden: hier + jetzt.

Schweizerischer Bundesrat (1938): Botschaft des Bundesrates an die Bundesversammlung über die Organisation und die Aufgaben der schweizerischen Kulturwahrung und Kulturwerbung (vom 9. Dezember 1938). Bundesblatt 90 (50, Bd. II), 985-1035, http://www.amtsdruckschriften.bar.admin.ch/view OrigDoc.do?id=10033812, 1.10.2018.

Sterne, Jonathan, Hg. (2012): The Sound Studies Reader. London/New York: Routledge.

Wiarda, Howard J. (2016): Political Culture, Political Science, and Identity Politics: An Uneasy Alliance. London/New York: Routledge. 